\title{
Methylene Blue for the Treatment of Intractable Pain From Oral Mucositis Related to Cancer Treatment: An Uncontrolled Cohort
}

\author{
Carlos J. Roldan, MD ${ }^{1,2}$; Matthew Chung, MD'; Lei Feng, $\mathrm{MS}^{3}$; and Eduardo Bruera, $\mathrm{MD}^{4}$
}

\begin{abstract}
Background: Oral mucositis is a complication of cancer therapy, causing severe pain that affects oral functioning, nutrition, and quality of life, as well as therapy nonadherence or dose-limiting toxicity. Anecdotal experience has suggested that methylene blue (MB) oral rinse may be an effective and safe treatment of this oral pain. Methods: To evaluate the efficacy and safety of MB oral rinse for the treatment of oral pain due to mucositis in patients with cancer, we retrospectively evaluated patients who experienced refractory pain despite conventional therapy. Results: We identified 281 patients who received $M B$ oral rinse. Most were receiving treatment for leukemia $(n=85$; $30.3 \%)$ and head and neck squamous cell carcinoma $(n=84 ; 29.9 \%)$. The most common treatments were radiation therapy alone $(n=108$; $38.4 \%$ ) and chemoradiation ( $n=86 ; 30.6 \%$ ). Median duration of symptoms was 14 days. Mean (SD) numeric rating scale pain scores were 7.7 (1.83; median, 8) before MB oral rinse and 2.51 (2.76; median, 2) after $\mathrm{MB}$ oral rinse $(P<.0001)$. Most patients achieved pain control within the first 3 doses. The effectiveness of $M B$ oral rinse was independent of patient age, sex, cancer type, cancer stage, MB dilution, and pain duration or baseline pain scores. The lowest response to treatment was reported in individuals with esophageal mucositis. Few patients experienced adverse effects of MB oral rinse $(n=13$; $4.6 \%) ; 10$ had a transient burning sensation, 2 had transient blue discoloration of the teeth and mouth, and 1 had increased pain. Conclusions: $\mathrm{MB}$ oral rinse is an effective and safe treatment for refractory pain from oral mucositis related to cancer treatment.
\end{abstract}

J Natl Compr Canc Netw 2021;19(5):521-527 doi: $10.6004 /$ jnccn.2020.7651

${ }^{1}$ Department of Pain Medicine, The University of Texas MD Anderson Cancer Center; ${ }^{2}$ McGovern Medical School at The University of Texas Health Science Center at Houston; and ${ }^{3}$ Department of Biostatistics, and ${ }^{4}$ Department of Palliative, Rehabilitation, and Integrative Medicine, The University of Texas MD Anderson Cancer Center, Houston, Texas.

\section{Background}

Oral mucositis (OM) is a painful inflammation and ulceration of the oral mucous membranes that affects millions of patients with cancer. It is a treatment-related toxicity associated with chemotherapy, radiation therapy, and hematopoietic stem cell transplantation (SCT). ${ }^{1}$ Oral lesions cause pain, resulting in dehydration, malnutrition, medication noncompliance, interruption in therapy, or increased risk of infection. Pain caused by OM may lead to impairment of oral function, which may require more aggressive supportive measures, including placement of a transcutaneous gastric feeding tube, parenteral nutrition, or frequent hospitalizations, exponentially increasing morbidity and cost. ${ }^{2}$

Lesions that develop after chemotherapy or during preparation for SCT occur approximately 3 to 5 days after treatment initiation. In contrast, postradiation OM exhibits a gradual progression and potentially lasts for weeks after completion of radiation therapy. Ulcerations caused by mucositis are typically nonuniform and can merge to form one large lesion. ${ }^{3}$ The ulcerations are not the result of an inflammatory process and the borders are not erythematous unless there is a concomitant infection. In addition, lesions tend to develop on less keratinized areas and therefore are rarely found on the dorsum of the tongue, gingiva, and hard palate. ${ }^{3}$ The pathophysiology of $\mathrm{OM}$ includes mucosal and epithelial damage secondary to endothelial injury in the lamina propria. ${ }^{4}$ Symptoms correlate with the severity of mucositis, fluctuating between local irritation and a burning sensation to severe debilitating pain.

Efforts have increased to prevent $\mathrm{OM}$ and histologically reverse oral lesions; however, pain is the major problem causing interruption of treatment and less favorable prognosis. ${ }^{5}$ Current strategies to manage $\mathrm{OM}$ include oral hygiene, prophylactic antimicrobials, selective diets to decrease bacterial growth, and avoidance of foods that exacerbate pain, in addition to topical therapies, including coatings, artificial saliva, and neutralizing 
solutions. Preventive agents such as palifermin, a keratinocyte growth factor, and amifostine, a free-radical scavenger, as well as low-level laser therapy have shown promising results but have varying levels of evidence for reducing the severity of mucositis; therefore, expert and empirical judgment have been the recommended approach in guidelines for managing mucositis. ${ }^{6-9}$

Managing oral pain has been the most substantial challenge in addressing OM. Conventional alternatives include use of topical anesthetics found in different mixes, compounded products, and oral rinses. Emerging options for pain control include oral doxepin rinse, ketamine mouthwash, bupivacaine lozenge, polyvinylpyrrolidonesodium hyaluronate gel, and CAM2028. These therapies have varied yet encouraging results but are not exempt from adverse effects and toxicity. ${ }^{10-15}$ To date, there is no standard efficacious therapy to prevent or treat OM-related pain in patients with cancer. ${ }^{16}$ Escalating doses of opiate analgesics remains a suboptimal but commonly used alternative. Unfortunately, many patients lack access to or cannot afford opioids, and concerns exist regarding opioid dependency and addiction-related drug diversion.

In the search for effective options to treat OM, novel topical and systemic agents have failed. More recently, studies have been conducted of methylene blue (MB), a partially liposoluble vital dye used for a variety of diagnostic and therapeutic indications. The structural composition of MB seems to affect different levels of the nociceptive pathways, and has been useful in reducing pain syndromes. It causes inhibition of nitric oxide synthetase, guanylyl cyclase, and histamine ${ }^{17}$; pain and analgesia have been shown to be in part modulated by guanylyl cyclase. A study by Salman et al ${ }^{18}$ demonstrated a decrease in pain intensity during induction of anesthesia when MB was first injected compared with placebo. A systematic review by Fransiska et al ${ }^{19}$ described a local analgesic effect of MB after anorectal surgery pain by temporarily disrupting the sensory nerve conduction.

Prior to this, MB had never been used to treat oral pain caused by mucositis secondary to cancer treatment. Thus, in 2017 we pilot-tested a diluted MB oral rinse to treat uncontrolled oral pain from cancer therapy. Our preliminary clinical data suggested that $\mathrm{MB}$ oral rinse was safe and effective for controlling pain in patients with this condition. ${ }^{20}$ We soon learned that providers within other specialties at The University of Texas MD Anderson Cancer Center had rapidly adopted our mix as a secondline treatment of OM in patients for whom conventional therapy had failed. Therefore, we initiated the current study to investigate the efficacy and safety of MB oral rinse for OM-related pain in patients receiving cancer treatment.

\section{Methods}

\section{Study Setting and Population}

This retrospective study was performed at our academic tertiary care cancer center and was approved by the Institutional Review Board of The University of Texas MD Anderson Cancer Center. No written informed consent from participants was required.

\section{Data Collection}

Study candidates were electronically identified by searching an institutional pharmacy database using the 2Know Request System for billing codes associated with a diagnosis of OM (ICD-9 and ICD-10 codes 99232, 99213, and 99214) between December 1, 2017, and December 1, 2019. From this search, we identified patients who were prescribed $\mathrm{MB}$ oral rinse to treat pain from OM. We then selected and reviewed the medical records of patients whose medication was dispensed to use.

\section{Selection and Description of Participants}

We considered patients of any age, sex, cancer diagnosis, and disease stage, and included all treatment modalities (chemotherapy, radiation therapy, SCT, surgery, and combinations). Pain intensity among patients with documented oral pain from cancer treatment-related OM was measured using a numeric rating scale (NRS; scores ranged from 1-10).

Patient information was stored in a secure database, and access to the database for secondary projects such as the current report required a separate approval by the Institutional Review Board, which was obtained.

\section{Procedures}

Patients with intractable oral pain from OM were instructed to use $\mathrm{MB}$ oral rinse. Various dilutions of $\mathrm{MB}$ were prescribed $(0.025 \%, 0.05 \%$, and $0.1 \%)$ and patients were instructed to swish, gargle, and spit every 6 hours until achieving pain control. The dilutions given seemed to be based on provider preference or pharmacy availability. Other modalities of pain management were not discontinued, including systemic analgesics and oral rinses; however, patients had not experienced sufficient pain relief despite use of these treatments.

\section{Outcome Measures}

We aimed to compare self-rated NRS scores of oral pain before treatment with $\mathrm{MB}$ oral rinse with those reported after treatment. If documentation was available, we also reviewed the morphine equivalent daily dose (MEDD) used, WHO grade of OM, and the location, nature, and duration of pain before treatment with $\mathrm{MB}$ oral rinse. In addition, we collected information about the duration of the analgesic effect experienced with the medication and any adverse effects reported. 


\section{Statistical Analysis}

Our plan was to include 300 patients in the retrospective study. With a sample size of 300, a 2-sided $95 \%$ confidence interval for the mean pain reduction would extend to 0.113 times the standard deviation from the observed mean based on the large-sample $z$ statistic (nQuery Advisor 7.0; Statsols). Continuous variables were summarized using descriptive statistics, including mean, standard deviation, median, and range. Continuous variables included patient age, pain medication reduction, NRS before and after treatment, duration of pain before treatment, duration of pain relief, duration of pain reduction after treatment, dose of $\mathrm{MB}$, and concentration of MB used for treatment. Categorical variables were tabulated by frequencies and the corresponding percentages, and included WHO grade before treatment, adverse effects of $\mathrm{MB}$ oral rinse, and patient sex. Chi-square or Fisher exact test was used to evaluate the association between categorical variables. Wilcoxon signed rank test was used to evaluate whether the change in pain score from before to after treatment was significantly different from zero $(P<.05$ indicated statistical significance). Wilcoxon rank sum test was used to evaluate the difference in a continuous variable between patient groups. A boxplot was generated as a visual aid to show the difference in the distribution of a continuous variable between or among patient groups. Statistical software SAS 9.4 (SAS Institute Inc.) and Splus 8.2 (TIBCO Software Inc) were used for all analyses.

\section{Results}

\section{Patient Characteristics}

We identified 350 adult patients who were prescribed MB oral rinse for oral pain from mucositis related to cancer therapy. Per pharmacy files, 31 individuals (all outpatient) could not afford the compounding charges from the pharmacy. In addition, 6 patients declined to use the medication and 32 medical records had inadequate documentation, precluding imputation of the data. The final study population consisted of 281 patients. All patients had documentation of actively receiving oral rinses, and most received concomitant opiate analgesics in oral or parenteral form.

The providers prescribing MB oral rinse were from several departments, including supportive care, emergency medicine, critical care, leukemia, lymphoma, sarcoma, breast cancer, radiation oncology, head/neck oncology, SCT, urology, and internal medicine.

\section{Demographic Characteristics}

Demographic information for the 281 patients included in the analysis is summarized in Table 1. Mean age was 55 years (range, 18-83 years), and there were 114 women

\section{Table 1. Patient Demographic Characteristics}

\begin{tabular}{|c|c|}
\hline Characteristic & n (\%) \\
\hline Patients, N & 281 \\
\hline Mean age (range), y & $55(18-83)$ \\
\hline \multicolumn{2}{|l|}{ Sex } \\
\hline Male & $167(59.4)$ \\
\hline Female & $114(40.6)$ \\
\hline \multicolumn{2}{|l|}{ Cancer diagnosis } \\
\hline Leukemia & $85(30.3)$ \\
\hline Head and neck SCC & $84(29.9)$ \\
\hline Lymphoma & $43(15.3)$ \\
\hline Germ cell & $13(5.0)$ \\
\hline MDS & $11(3.9)$ \\
\hline Multiple myeloma & $10(3.6)$ \\
\hline Gastrointestinal & $9(3.2)$ \\
\hline Sarcoma & $6(2.1)$ \\
\hline Other & $20(7.1)$ \\
\hline Cancer therapy, $\mathrm{N}$ & 261 \\
\hline Radiation therapy & $114(43.7)$ \\
\hline Chemoradiation & $91(34.9)$ \\
\hline Stem cell transplantation & $3(1.1)$ \\
\hline Chemotherapy alone & $39(15.0)$ \\
\hline Surgery and radiation & $14(5.4)$ \\
\hline Mean duration of pain $( \pm S D) /$ median, days & $23.31( \pm 38.34) / 14$ \\
\hline
\end{tabular}

Abbreviations: MDS, myelodysplastic syndromes; SCC, squamous cell carcinoma.

and 167 men. Ethnicity was not considered relevant. The most common diagnoses included leukemia $(n=85$; $30.3 \%)$ and head and neck squamous cell carcinoma $(\mathrm{n}=84 ; 29.9 \%)$. Most patients were actively undergoing cancer therapy $(\mathrm{n}=261 ; 92.9 \%)$. The most common therapeutics used were radiation alone $(n=108 ; 38.4 \%)$ and chemoradiation $(n=86 ; 30.6 \%)$. Median duration of mucositis-related pain was 14 days.

\section{Clinical Characteristics}

Clinical patient characteristics are summarized in Table 2. Most patients ( $\mathrm{n}=207 ; 73.7 \%)$ had painful mucosal lesions of the oral and esophageal structures; the oral mucosa was the most commonly reported $(n=188 ; 66.9 \%)$. Most commonly, pain description was burning $(n=155$; $70.1 \%)$. Only 126 patients had WHO grade of mucositis documented, with most grade III $(\mathrm{n}=82 ; 65.1 \%)$. The MB dilution used most commonly was $0.025 \%(n=179$; $63.7 \%)$.

\section{Efficacy of MB}

Among the 281 patients, 243 had pain scores reported both before and after MB oral rinse therapy. Mean (SD) 


\section{Table 2. Patient Clinical Characteristics}

\begin{tabular}{|c|c|}
\hline Characteristic & n (\%) \\
\hline Patients, N & 281 \\
\hline \multicolumn{2}{|l|}{ Location of pain } \\
\hline Oral mucosa & $188(66.9)$ \\
\hline Sublingual & $86(30.6)$ \\
\hline Oropharynx & $26(9.3)$ \\
\hline Esophageal & $23(8.2)$ \\
\hline Soft palate & $21(7.5)$ \\
\hline Inner lips & $12(4.3)$ \\
\hline Multiple locations & $207(73.7)$ \\
\hline Pain description, $\mathrm{N}$ & 221 \\
\hline Oral burning & $155(70.1)$ \\
\hline Aching & $56(25.3)$ \\
\hline Sharp & $10(4.5)$ \\
\hline Cutting blades & $27(12.2)$ \\
\hline WHO grade, $\mathrm{N}$ & 126 \\
\hline IV & $14(11.1)$ \\
\hline III & $82(65.1)$ \\
\hline ॥ & $30(23.8)$ \\
\hline \multicolumn{2}{|l|}{ Adverse effects } \\
\hline Transient burning sensation & $10(3.6)$ \\
\hline Transient discoloration & $2(0.7)$ \\
\hline Increased pain & $1(0.4)$ \\
\hline \multicolumn{2}{|l|}{ MB dilution used } \\
\hline $0.025 \%$ & $179(63.7)$ \\
\hline $0.05 \%$ & $90(32.0)$ \\
\hline $0.1 \%$ & $12(4.2)$ \\
\hline
\end{tabular}

Abbreviation: $\mathrm{MB}$, methylene blue.

NRS pain score before MB oral rinse therapy was 7.7 (1.83; median, 8) and after therapy was 2.51 (2.76; median, 2). Mean (SD) NRS pain score reduction was 5.12 (2.97; median, 5), and the Wilcoxon signed rank test indicated that the change in pain score from before to after treatment was significantly different $(P<.0001$; Figure 1$)$. Among the 243 patients, 205 (84.4\%) had a baseline NRS pain score $\geq 7$ and 38 had a baseline NRS pain score $<7$ (15.6\%). The difference in pain score change among the different $\mathrm{MB}$ dilution groups was not statistically significant $(P=.66$; Figure 2$)$.

The maximum pain relief was reported within minutes of the first dose in most patients $(n=124 ; 58.5 \%)$; the remaining patients required several doses—up to 9 doses (48-72 hours) - to achieve sufficient pain relief (Table 3). Although more than half of the patients in whom pain scores were reported achieved sustained pain relief after the first dose $(n=131 ; 55.5 \%)$, others reported pain recurrence between 4 and 8 hours $(n=58 ; 24.6 \%)$. However,

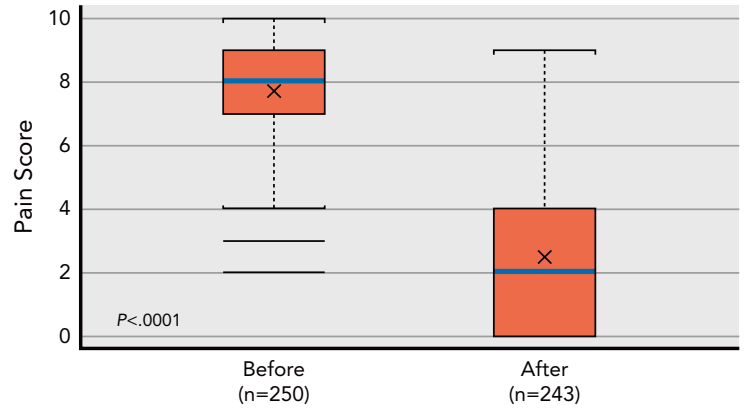

Figure 1. NRS pain scores before and after treatment with MB oral rinse. Bar graph shows the mean ( $\mathrm{X}$ inside the bar), median (white bar inside the box), and outliers (horizontal lines beyond the end of the vertical dotted lines) of the pain scores before (left) and after (right) treatment with $\mathrm{MB}$ oral rinse. Pain reduction was significantly different from zero according to the Wilcoxon signed rank test $(P<.0001)$.

Abbreviations: MB, methylene blue; NRS, numeric rating scale.

the subsequent intensity was reported at lower scores than at baseline, which suggested an accumulative analgesic effect up to complete control within 3 days. In the remaining cases, recurrence was not clearly documented.

Many patients had documented multisite pain beyond $\mathrm{OM}$; therefore, conclusions regarding the effect of MB oral rinse on MEDD could not be drawn.

Oral functioning (ability to talk, chew, and swallow) before and after MB was inconsistently documented, and 5 patients had already had a gastric tube placed before using the MB oral rinse.

\section{Association Between Demographic Characteristics and MB Efficacy}

Reduction in NRS pain scores during a specified time frame was documented in 212 patients, who were divided into interval groups according to reduction in pain scores: $>5,2$ to 5 , and $<2$ (Table 3 ). Most patients whose pain scores were reported achieved pain reduction of $\geq 5$ on the NRS ( $n=138 ; 65.0 \%$ ), and only 11 patients (5.2\%) reported pain reduction of $<2$. The difference in pain score change among age groups was not statistically significant $(P=.48)$. Similarly, the difference in pain score change between sexes was insignificant $(P=.1853)$. Patients with esophageal lesions reported lower pain score changes compared with others $(P<.05)$.

\section{Adverse Effects}

Few mild and transient adverse effects were reported $(n=13 ; 4.6 \%$; Table 2). A total of 10 patients experienced an oral burning sensation during first-time use, with 1 subsequently discontinuing therapy. Two patients reported transient blue discoloration of the teeth and mouth, which resolved with oral hygiene. One patient experienced increased pain during MB oral rinse use, resulting in discontinuation of therapy. 


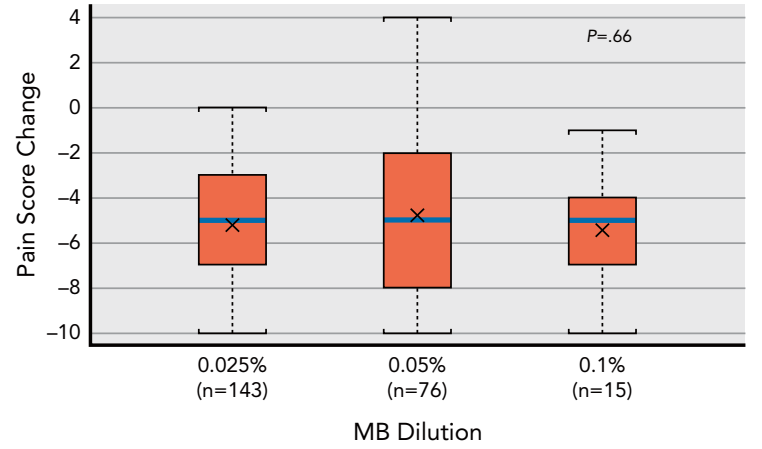

Figure 2. NRS pain scores after treatment with MB oral rinse at 3 different dilutions. Bar graph shows the mean ( $X$ inside the bar) and median (white bar inside the box) change in pain score after treatment with $\mathrm{MB}$ oral rinse at $0.025 \%, 0.05 \%$, and $0.1 \%$ dilution. The graph shows no statistical difference among the groups according to the Wilcoxon rank sum test $(P=.66)$.

Abbreviations: $\mathrm{MB}$, methylene blue; NRS, numeric rating scale.

\section{Discussion}

In 2017, we conducted a pilot clinical test of MB oral rinse $(0.05 \%)$ in 5 patients with severe oral pain from mucositis related to cancer therapy. ${ }^{20}$ Based on promising results, a phase II prospective trial of $\mathrm{MB}$ oral rinse was designed and implemented. It included 4 treatment arms: 3 using MB oral rinse at $0.025 \%, 0.05 \%$, and $0.1 \%$, respectively, and 1 using conventional therapy alone (ClinicalTrials.gov identifier: NCT03469284). Although data were being collected, preliminary results of safety and efficiency became visible to providers from different departments within our institution who adopted our protocol for clinical use (including MB doses and frequency of use). Because of the increasing number of documented prescriptions of MB oral rinse, we were able to collect sufficient data for the current study. Our results support the use of MB oral rinse as an easy-to-use, safe, and efficient therapy for intractable pain associated with $\mathrm{OM}$ in patients with cancer.

When administered orally, MB is absorbed through the gastrointestinal tract, with peak plasma concentration occurring in 1 to 2 hours. ${ }^{21}$ However, this concentration is 100 -fold less than concentrations achieved after intravenous administration of an equivalent dose. ${ }^{22}$ This significant difference is attributable to first-pass metabolism in the liver after oral administration. ${ }^{23}$ Therefore, the pharmacokinetics of MB suggest that if diluted to $0.025 \%$ to $0.1 \%$, and if strictly used as an oral rinse, the plasma concentration of $\mathrm{MB}$ absorbed is potentially negligible and therefore exempt from pharmacologic interactions or toxicity. In this study, no toxicities or medication interactions were reported, suggesting that $M B$ is safe when used as an oral rinse. If accidentally ingested, $\mathrm{MB}$ is metabolized to leukomethylene blue and excreted primarily in urine, turning the urine a blue-green color. ${ }^{24}$ The terminal half-life is estimated to be 5.25 hours. ${ }^{25}$ This is our rationale for prescribing its use only every 6 hours, to avoid any remote chance of systemic accumulation.

The only on-label use of MB approved by the FDA is for the treatment of methemoglobinemia, for which $\mathrm{MB}$ is administered intravenously. ${ }^{26}$ Through this route, MB has also been used for the management of vasoplegia (a high-output shock state with poor systemic vascular resistance, a common occurrence in patients undergoing cardiothoracic surgery) refractory to catecholamines, and as an adjuvant to treat cyanide toxicity. ${ }^{27,28}$ Oral MB has been used in the treatment of malaria in sub-Saharan Africa $^{21}$ and for the treatment of ifosfamide-induced encephalopathy. ${ }^{29}$ For diagnostic purposes, MB is used for tissue staining to help detect various gastrointestinal pathologies. $^{30-32}$ Similarly, intra-articular MB is used to establish the integrity of the capsule of the knee joint in trauma patients. ${ }^{33}$ When coupled with visible light therapy, antimicrobial properties of MB are used to inactivate lipid-enveloped viruses. ${ }^{31}$ Furthermore, as a $2 \%$ topical solution, $\mathrm{MB}$ has been effective in treating onychomycosis. ${ }^{34}$ Lastly, a $0.1 \%$ topical solution of MB in combination with fluorescent light has been used in the treatment of genital herpes simplex infections. ${ }^{35}$

\section{Table 3. Peak Pain Reduction Per Time and Dose Received of MB Oral Rinse $(\mathbf{N}=212)$}

\begin{tabular}{|c|c|c|c|c|}
\hline $\begin{array}{l}\text { Time of Peak } \\
\text { Pain Relief }\end{array}$ & $\begin{array}{c}\text { Any Pain Reduction } \\
\mathrm{n}(\%)\end{array}$ & $\begin{array}{c}\text { Pain Reduction }>5 \text { on NRS } \\
n(\%)\end{array}$ & $\begin{array}{c}\text { Pain Reduction 2-5 on NRSa } \\
\text { n (\%) }\end{array}$ & $\begin{array}{c}\text { Pain Reduction }<2 \text { on NRSa } \\
n(\%)\end{array}$ \\
\hline $0 \mathrm{~h}$ (dose 1) & $124(58.5)$ & $76(61.3)$ & $41(33.1)$ & $7(5.6)$ \\
\hline 2-12 h (dose 1-2) & $11(5.2)$ & $9(81.8)$ & $2(18.2)$ & $0(0.0)$ \\
\hline 12-24 h (dose 2-4) & $52(24.5)$ & $36(69.2)$ & $14(26.9)$ & $2(3.8)$ \\
\hline 24-36 h (dose 4-6) & $1(0.5)$ & $1(100.0)$ & $0(0.0)$ & $0(0.0)$ \\
\hline $48-72 \mathrm{~h}$ (dose 8-10) & $8(3.8)$ & $7(87.5)$ & $0(0.0)$ & $1(12.5)$ \\
\hline
\end{tabular}

Abbreviations: MB, methylene blue; NRS, numeric rating scale.

${ }^{\text {a }}$ Percentages in these columns refer to the number of patients who reported any level of peak pain reduction at that time point. 
Systemic use of MB is contraindicated in pregnant and nursing women, patients with glucose-6-phosphate dehydrogenase deficiency, and in individuals allergic to it. ${ }^{36} \mathrm{MB}$ can cause hemolytic anemia in patients with glucose-6-phosphate dehydrogenase deficiency because of its decreased ability to metabolize MB. ${ }^{18}$ In addition, because $\mathrm{MB}$ is a monoamine oxidase inhibitor, it must be used with caution in patients receiving other proserotonin medications. ${ }^{37}$ In addition, MB can stain tissue, skin, clothing, or whatever else it may come in contact with.

MB seems to be a long-term inhibitor of peripheral axons and an antioxidant and anti-inflammatory agent, which is why it is a useful analgesic. ${ }^{38}$ When used as an oral rinse, $\mathrm{MB}$ is thought to denature free nociceptive nerve endings, inhibit the nitric oxide inflammatory pathway, and block $N$-methyl-D-aspartate receptors. ${ }^{39-41}$ In this study, the lower pain response in patients with esophageal lesions supports the need for direct contact between $\mathrm{MB}$ and the compromised mucosa, given that it was not swallowed.

Unlike other oral rinses containing topical anesthetics, MB does not cause local anesthesia, which is manifested as intraoral numbness. Thus, MB does not alter the taste of ingested meals. In addition, MB seems to have an accumulative analgesic effect, as opposed to the short-lasting pain relief encountered with other rinses compounded with local anesthetics.

Given that the MB available in our setting is only in the form of vials for intravenous use, high cost is a limitation to its use. In addition, compounding charges are a tremendous barrier for many patients, even those who have health insurance. Surprisingly, while visiting a developing country we found MB easily accessible and inexpensive at local pharmacies in a nonintravenous form. It was tested by local providers with results similar to those we have reported.

This study had a few limitations. Inadequacies in the data acquisition could not be addressed due to the retrospective nature of the study. In addition, a discrepancy in the size of the comparison groups could not be reconciled. Lastly, we could not objectively address MEDD before and after MB oral rinse therapy.

\section{Conclusions}

$\mathrm{MB}$ oral rinse is a promising therapy for pain from $\mathrm{OM}$ related to cancer therapy, although long-term benefits and risks have yet to be explored. MB is a low-risk, efficient, easy-to-use treatment for refractory pain in $\mathrm{OM}$ related to cancer therapy, and potentially accessible to patients of all socioeconomic backgrounds in the absence of compounding charges.

Submitted July 21, 2020; accepted for publication September 3, 2020. Published online January 4, 2021.

Author contributions: Study concept: Roldan, Bruera. Data curation: Roldan, Chung. Investigation: Roldan. Methodology: Roldan. Project administration: Roldan. Supervision: Roldan. Formal analysis: Feng. Writing-original draft: Roldan. Writing_review and editing: All authors.

Disclosures: The authors have disclosed that they have not received any financial consideration from any person or organization to support the preparation, analysis, results, or discussion of this article.

Correspondence: Carlos J. Roldan, MD, Department of Pain Medicine, The University of Texas MD Anderson Cancer Center, Unit 409, 1515 Holcombe Boulevard, Houston, TX 77030. Email: croldan@mdanderson.org

\section{References}

1. Keefe DM, Schubert MM, Elting LS, et al. Updated clinical practice guidelines for the prevention and treatment of mucositis. Cancer 2007; 109:820-831.

2. Elting LS, Keefe DM, Sonis ST, et al. Patient-reported measurements of oral mucositis in head and neck cancer patients treated with radiotherapy with or without chemotherapy: demonstration of increased frequency, severity, resistance to palliation, and impact on quality of life. Cancer 2008;113:2704-2713.

3. Sonis S. Oral Mucositis: Pocket Books for Cancer Supportive Care. London, UK: Springer Healthcare; 2012. https://doi.org/10.1007/9781-907673-46-7

4. Sonis ST. Pathobiology of oral mucositis: novel insights and opportunities. J Support Oncol 2007;5(Suppl 4):3-11.

5. Roldan CJ, Chai T, Erian J, et al. Oral pain associated with cancer therapy, a pain medicine perspective. Pain Manag 2018;8:487-493.

6. Lalla RV, Bowen J, Barasch A, et al. MASCC/ISOO clinical practice guidelines for the management of mucositis secondary to cancer therapy. Cancer 2014;120:1453-1461

7. Kanuga S. Cryotherapy and keratinocyte growth factor may be beneficial in preventing oral mucositis in patients with cancer, and sucralfate is effective in reducing its severity. J Am Dent Assoc 2013;144:928-929.

8. Ferreira B, da Motta Silveira FM, de Orange FA. Low-level laser therapy prevents severe oral mucositis in patients submitted to hematopoietic stem cell transplantation: a randomized clinical trial. Support Care Cancer 2016;24:1035-1042.
9. Bensinger W, Schubert M, Ang KK, et al. NCCN Task Force Report: prevention and management of mucositis in cancer care. J Natl Compr Canc Netw 2008;6(Suppl 1):S1-21.

10. Ryan AJ, Lin F, Atayee RS. Ketamine mouthwash for mucositis pain J Palliat Med 2009;12:989-991.

11. Mogensen S, Sverrisdóttir E, Sveinsdóttir K, et al. Absorption of bupivacaine after administration of a lozenge as topical treatment of pain from oral mucositis. Basic Clin Pharmacol Toxicol 2017;120:71-78.

12. Buchsel PC. Polyvinylpyrrolidone-sodium hyaluronate gel (Gelclair): a bioadherent oral gel for the treatment of oral mucositis and other painful oral lesions. Expert Opin Drug Metab Toxicol 2008;4:1449-1454.

13. Barber C, Powell R, Ellis A, et al. Comparing pain control and ability to eat and drink with standard therapy vs Gelclair: a preliminary, double centre, randomised controlled trial on patients with radiotherapy-induced oral mucositis. Support Care Cancer 2007;15:427-440

14. Vokurka S, Skardova J, Hruskova R, et al. The effect of polyvinylpyrrolidone-sodium hyaluronate gel (Gelclair) on oral microbial colonization and pain control compared with other rinsing solutions in patients with oral mucositis after allogeneic stem cells transplantation. Med Sci Monit 2011;17:CR572-576.

15. Hadjieva T, Cavallin-Stahl E, Linden M, Tiberg F. Treatment of oral mucositis pain following radiation therapy for head-and-neck cancer using a bioadhesive barrier-forming lipid solution. Support Care Cancer 2014; 22:1557-1562. 
16. Rubenstein EB, Peterson DE, Schubert $M$, et al. Clinical practice guidelines for the prevention and treatment of cancer therapy-induced oral and gastrointestinal mucositis. Cancer 2004;100(9 Suppl):2026-2046.

17. Friebe A, Koesling D. Regulation of nitric oxide-sensitive guanylyl cyclase. Circ Res 2003;93:96-105.

18. Salman AE, Salman MA, Saricaoglu $F$, et al. Pain on injection of propofol: a comparison of methylene blue and lidocaine. J Clin Anesth 2011;23:270-274.

19. Fransiska D, Jeo WS, Moenadjat $Y$, et al. Methylene blue effectiveness as local analgesic after anorectal surgery: a literature review. Adv Med 2017; 2017:3968278.

20. Roldan CJ, Nouri K, Chai T, et al. Methylene blue for the treatment of intractable pain associated with oral mucositis. Pain Pract 2017;17:1115-1121.

21. Rengelshausen J, Burhenne J, Fröhlich M, et al. Pharmacokinetic interaction of chloroquine and methylene blue combination against malaria. Eur J Clin Pharmacol 2004;60:709-715.

22. Walter-Sack I, Rengelshausen J, Oberwittler $\mathrm{H}$, et al. High absolute bioavailability of methylene blue given as an aqueous oral formulation. Eur J Clin Pharmacol 2009;65:179-189.

23. DiSanto AR, Wagner JG. Pharmacokinetics of highly ionized drugs. I. Methylene blue-whole blood, urine, and tissue assays. J Pharm Sci 1972; 61:598-602.

24. McDonagh EM, Bautista JM, Youngster I, et al. PharmGKB summary: methylene blue pathway. Pharmacogenet Genomics 2013;23:498-508.

25. Peter C, Hongwan D, Küpfer A, et al. Pharmacokinetics and organ distribution of intravenous and oral methylene blue. Eur J Clin Pharmacol 2000;56:247-250

26. Methylene blue IV injection [product information]. Decatur, IL: Taylor Pharmaceuticals; 2006.

27. Hencken L, To L, Ly N, et al. Serotonin syndrome following methylene blue administration for Vasoplegic syndrome. J Card Surg 2016;31:208-210.

28. Gennaro AR. Remington's Pharmaceutical Sciences, 18th ed. Easton, PA: Mack Printing Company; 1990.

29. Turner AR, Duong CD, Good DJ. Methylene blue for the treatment and prophylaxis of ifosfamide-induced encephalopathy. Clin Oncol (R Coll Radiol) 2003;15:435-439.
30. Fennerty MB, Sampliner RE, McGee DL, et al. Intestinal metaplasia of the stomach: identification by a selective mucosal staining technique. Gastrointest Endosc 1992;38:696-698.

31. Masaki T, Sheffield JP, Talbot IC, et al. Non-polypoid adenoma of the large intestine. Int J Colorectal Dis 1994;9:180-183.

32. Kiesslich R, Burg J, Vieth $M$, et al. Confocal laser endoscopy for diagnosing intraepithelial neoplasias and colorectal cancer in vivo. Gastroenterology 2004;127:706-713.

33. Metzger P, Carney J, Kuhn K, et al. Sensitivity of the saline load test with and without methylene blue dye in the diagnosis of artificial traumatic knee arthrotomies. J Orthop Trauma 2012;26:347-349.

34. Lambrecht B, Mohr H, Knüver-Hopf J, et al. Photoinactivation of viruses in human fresh plasma by phenothiazine dyes in combination with visible light. Vox Sang 1991;60:207-213.

35. Souza LW, Souza SV, Botelho AC. Distal and lateral toenail onychomycosis caused by Trichophyton rubrum: treatment with photodynamic therapy based on methylene blue dye. An Bras Dermatol 2014;89:184-186.

36. Chang TW, Fiumara N, Weinstein L. Letter: methylene blue and light therapy for herpes simplex. Arch Dermatol 1975;111:265-266.

37. Methylene blue. Wolters Kluwer at Lexicomp, 2018. Accessed December 16, 2019. Available at: https://online.lexi.com/lco/action/doc/retrieve/ docid/patch_f/7279

38. Faber P, Ronald A, Millar BW. Methylthioninium chloride: pharmacology and clinical applications with special emphasis on nitric oxide mediated vasodilatory shock during cardiopulmonary bypass. Anaesthesia 2005;60: 575-587. https://doi.org/10.1111/j.1365-2044.2005.04185.x

39. Baron R, Binder A, Wasner G. Neuropathic pain: diagnosis, pathophysiological mechanisms, and treatment. Lancet Neurol 2010;9:807-819. https://doi.org/10.1016/S1474-4422(10)70143-5

40. Mentes BB, Akin M, Leventoglu S, et al. Intradermal methylene blue injection for the treatment of intractable idiopathic pruritus ani: results of 30 cases. Tech Coloproctol 2004;8:11-14. https://doi.org/10.1007/ s10151-004-0043-y

41. Tan KY, Seow-Choen F. Methylene blue injection reduces pain after latera anal sphincterotomy. Tech Coloproctol 2007;11:68-69. 